\title{
Perturbing resistance: a network perspective
}

\author{
Levesque, Mitchell P ; Dummer, Reinhard ; Beerenwinkel, Niko
}

\begin{abstract}
The recent convergence of high-dimensional molecular datasets with small-molecule inhibitor pipelines allows for selective targeting of aberrantly regulated pathways in many cancer types. But intratumor heterogeneity, paradoxical activation, intrinsic or acquired therapeutic resistance, and non-linear pathway interactions confound most simple targeting strategies(Widmer et al., 2015). For instance, MAPK signaling is activated by hot-spot mutations in BRAF, which are found in about 40-50\% of melanoma cases. This article is protected by copyright. All rights reserved.
\end{abstract}

DOI: https://doi.org/10.1111/pcmr.12431

Posted at the Zurich Open Repository and Archive, University of Zurich

ZORA URL: https://doi.org/10.5167/uzh-116953

Journal Article

Accepted Version

Originally published at:

Levesque, Mitchell P; Dummer, Reinhard; Beerenwinkel, Niko (2015). Perturbing resistance: a network perspective. Pigment Cell Melanoma Research, 29(1):5-7.

DOI: https://doi.org/10.1111/pcmr.12431 
Perturbing resistance: a network perspective

\author{
Mitchell P. Levesque ${ }^{1^{*}}$, Reinhard Dummer ${ }^{1}$, Niko Beerenwinkel ${ }^{2}$
}

(1) University of Zürich, University of Zürich Hospital, Gloriastrasse 31, Zürich Switzerland CH-8091

(2) Swiss Federal Institute of Technology Zürich, Computational Biology Group, Office 8.40, Mattenstrasse 26, Basel Switzerland, $\mathrm{CH}-4058$

${ }^{*}$ Corresponding author: Mitchell.levesque@usz.ch

The recent convergence of high-dimensional molecular datasets with small-molecule inhibitor pipelines allows for selective targeting of aberrantly regulated pathways in many cancer types. But intra-tumor heterogeneity, paradoxical activation, intrinsic or acquired therapeutic resistance, and nonlinear pathway interactions confound most simple targeting strategies(Widmer et al., 2015).

For instance, MAPK signaling is activated by hot-spot mutations in BRAF, which are found in about $40-50 \%$ of melanoma cases. Although the BRAF-inhibitors vemurafenib and dabrafenib have been incredibly effective in the clinic, sustained long-term responses to single-agent therapy have been rare. The combination of BRAF and MEK inhibitors, such as trametinib and cobimetinib, has prolonged response duration and improved overall survival, but most patients still progress after $\sim 1$ year of therapy (Robert et al., 2015). Thus, the mechanisms of therapeutic resistance are under intense investigation at the moment, with molecularly driven rational combinations of more than two targeted therapies already in clinical trials. The challenge, however, is in using extensive molecular data to identify who might benefit from which drug combinations or treatment schedules.

The Systems Biology paradigm offers a solution to this problem by generating more complex and testable models of the dynamic network properties of cells(Levesque and Benfey, 2004). An excellent example was recently published in the open-access journal eLife. The authors followed previous work in which they had screened 149 melanoma cell lines to identify BRAF ${ }^{\mathrm{V} 600 \mathrm{E}}$ mutated cells that were resistant to inhibition of the downstream MEK protein(Xing et al., 2012). Thus, although these cells had a BRAF-dependent activation of the MAPK pathway, they did not require MEK phosphorylation for growth. Interestingly, they demonstrated in that earlier publication that a combined loss of the RB1 and PTEN proteins conferred this MEK-resistance. This functional characterization of the RAFi-resistant cell line SkMel-133 was the basis for the recent paper in eLife in which Korkut et al. measured 143 proteomic and phenotypic variables in 89 systematic perturbation conditions of this one cell line. These data were then used to construct comprehensive quantitative models of proteomic and phenotypic outcomes under any perturbation.

Biologists have always used perturbations, or interventions, to study the behavior of biological systems. The term "perturbation biology" does justice to the fact that recent technological advances allow for large-scale perturbation experiments in which many different combinatorial perturbations are performed and the read-out includes high-dimensional molecular profiles. While interventional data is statistically very powerful, and in principle allows even for causal inference, in practice, it remains challenging to analyze data from large-scale perturbation experiments.

Korkut el al. have made an attempt to scale up and to improve their previously described perturbation biology in order to predict new perturbations against drug resistant melanoma cells that could be implemented by combinations of drugs(Molinelli et al., 2013). Their approach is based on a system of non-linear ordinary differential equations that describe the dynamic molecular interactions of the components of a signaling pathway. For the most challenging task of inferring the topology of the signaling pathway, they developed an approximate but computationally very efficient algorithm based on belief propagation, a general strategy to compute marginal probabilities in probabilistic graphical models.

While previously the authors tried to learn the network topology from the perturbation data from scratch, in their current approach they make use of signaling databases that collect and summarize existing knowledge. They construct a network distribution that reflects our a priori knowledge of the network topology, i.e., or knowledge before seeing the new perturbation data. The network is then learned by penalizing not only poor fit to the data but also increased complexity of the model and deviation from the prior network. This Bayesian approach has proven to be successful in many biological data analysis and interpretation tasks. Here, the authors introduce a flexible mathematical description of the prior network knowledge, and they present an algorithm to extract directed signaling paths from signaling databases given a list of observed (phospho)proteins and their molecular activation states. Their careful construction of the prior signaling network distribution is more finegrained than previous approaches and takes into account multiple phosphorylation sites per protein and lack of tissue specificity in databases. 
Importantly, the authors did not stop at network modeling, but used the learned model for predicting the effect of previously unobserved perturbations. Specifically, they tested the generated hypothesis that co-targeting of c-Myc and MAPK proteins would overcome BRAFi-resistance in SkMel-133 cells. They demonstrated that JQ1, which is a BET bromodomain inhibitor that reduces c-Myc expression, abrogated SkMel-133 growth in combination with BRAFi in vitro. Moreover, they examined one of the key predictions of their network model, which was that the SkMel-133 melanoma cells should arrest in G1 upon single or combination treatment with c-Myc and the MAPK targets. Indeed, they could confirm the strongest cell cycle arrest in the MEKi/JQ1 combination with $92 \%$ of cells arresting in G1. They suggest that such drug combinations may target potentially resistant subpopulations and thereby delay the onset of therapeutic resistance in patients.

Reconstructing complex molecular pathways that include feedback modules and non-linear interactions requires such a "perturbation biology" approach, since network wiring may be somewhat context and cell-type specific. However, this paper also illustrates how difficult it will be to implement in a clinical setting. For instance, it has been suggested that there is a long-tail of resistance mechanisms, and it is still an open question to what degree these will converge on a common set of pathways that could be targetable with the "upstream-downstream" strategy presented by Korkut et al. The authors conducted all of their experiments with a single cell line that had a genotype and expression pattern that were atypical of the 149 cells that were originally screened. In addition, the experiments that produced the data on which the network inference was based would likely have a large impact on the final drug predictions, since the RPPA assay, the phenotype measurements, and the inhibitor panels used in this study represent only a small proportion of the proteomic, phenotypic, and inhibitor spaces that must be explored for better pathway targeting.

Thus, it is unclear how generalizable their findings are for other melanoma cells in vitro and in vivo. In fact, as mentioned in the paper, the actual drug combination they identified would probably not be effective in actual melanoma patients since the JQ1 inhibitor has rather poor in vivo solubility and efficacy. It is possible that other c-Myc inhibitors will be more effective in vivo, but this problem illustrates the challenge of translating even very sophisticated in silico and in vitro results into clinically relevant treatment strategies.

Modern molecular biology is providing huge genomics, epigenomics, and proteomics data sets that need to be integrated in the daily management of patients. These data contain a lot of irrelevant noise with little predictive power, as well as some clinically meaningful signals. Based on the complexity of biological systems, medical bioinformatics and computational biology strategies must be established that support physicians in decision making and facilitate a rapid prediction and evaluation of a treatment's clinical efficacy. This requires close interactions between researchers in many fields to develop the experimental and mathematical tools to meet this challenge. While Korkut et al. have taken a step forward in translating the perturbation biology approach to the clinic, there is still a long way to go to understand how best to target such complex adaptive systems in a way that can directly benefit melanoma patients today.

\section{Coverage of:}

"Perturbation biology nominates upstream-downstream drug combinations in RAF inhibitor resistant melanoma cells". Korkut et al.

\section{References}

Levesque, M. P., and Benfey, P. N. (2004). Systems biology. Curr Biol 14, R179-80.

Molinelli, E. J., Korkut, A., Wang, W., Miller, M. L., Gauthier, N. P., Jing, X., Kaushik, P., He, Q., Mills, G., Solit, D. B., et al. (2013). Perturbation biology: inferring signaling networks in cellular systems. PLoS Comput Biol 9, e1003290.

Robert, C., Karaszewska, B., Schachter, J., Rutkowski, P., Mackiewicz, A., Stroiakovski, D., Lichinitser, M., Dummer, R., Grange, F., Mortier, L., et al. (2015). Improved overall survival in melanoma with combined dabrafenib and trametinib. N Engl J Med 372, 30-9.

Widmer, D. S., Eichhoff, O. M., Dummer, R., and Levesque, M. P. (2015). Melanoma's next top model, it is in the air. Exp Dermatol 24, 659-60.

Xing, F., Persaud, Y., Pratilas, C. A., Taylor, B. S., Janakiraman, M., She, Q. B., Gallardo, H., Liu, C., Merghoub, T., Hefter, B., et al. (2012). Concurrent loss of the PTEN and RB1 tumor suppressors attenuates RAF dependence in melanomas harboring (V600E)BRAF. Oncogene $31,446-57$. 